\title{
Stochastic Self-Similar and Fractal Universe
}

\author{
G.Iovane* \\ Dipartimento di Ingegneria dell'Informazione e Matematica Applicata, \\ Universitá di Salerno, Italy. \\ Gruppo Nazionale di Fisica Matematica, Italy \\ E.Laserra \\ Dipartimento di Matematica e Informatica, Universitá di Salerno, Italy \\ Gruppo Nazionale di Fisica Matematica, Italy \\ F.S.Tortoriello \\ Universitá di Salerno, Italy \\ Gruppo Nazionale di Fisica Matematica, Italy.
}

Last changed 20.07.03

\begin{abstract}
The structures formation of the Universe appears as if it were a classically self-similar random process at all astrophysical scales. An agreement is demonstrated for the present hypotheses of segregation with a size of astrophysical structures by using a comparison between quantum quantities and astrophysical ones. We present the observed segregated Universe as the result of a fundamental self-similar law, which generalizes the Compton wavelength relation. It appears that the Universe has a memory of its quantum origin as suggested by R.Penrose with respect to quasi-crystal. A more accurate analysis shows that the present theory can be extended from the astrophysical to the nuclear scale by using generalized (stochastically) self-similar random process. This transition is connected to the relevant presence of the electromagnetic and nuclear interactions inside the matter. In this sense, the presented rule is correct from a subatomic scale to an astrophysical one. We discuss the near full agreement at organic cell scale and human scale too. Consequently the Universe, with its structures at all scales (atomic nucleus, organic cell, human, planet, solar system, galaxy, clusters of galaxy, super clusters of galaxy), could have a fundamental quantum reason. In conclusion, we analyze the spatial dimensions of the objects in the Universe as well as spacetime dimensions.
\end{abstract}

*iovane@diima.unisa.it 
The result is that it seems we live in an El Naschie's E infinity Cantorian spacetime; so we must seriously start considering fractal geometry as the geometry of nature, a type of arena where the laws of physics appear at each scale in a self-similar way as advocated long ago by the Swedish school of astrophysics.

\section{Introduction}

What is the geometry of the universe? Has the universe a memory of its quantum and relativistic origin?

In 1965 Sakharov indicated that quantum primordial fluctuations should have expanded towards the present epoch leading first to classical energy-density perturbations and, after the decoupling from the cosmological background, to the observed galaxies, clusters and superclusters of galaxies [1].

A relevant contribution was given by L.Nottale starting from 1993. In many papers he extends Einstein's principle of relativity to scale transformations in the framework of the theory of scale relativity. In particular, he showed that a continuous but non differentiable space-time is necessarily fractal [2], 3], 4, 5. In this work, we present a complementary approach starting from the wellknown Random Walk equation or Brownian motion relation that was firstly used by Eddington 6], 7, [8. Following this line we arrive at a self-similar universe; which was firstly considered by the Swedish Astronomers Charlier [9]. By taking into account a generalization of Compton wavelength rule, the model realizes a segregated universe, where the sizes of astrophysical structures can fit the observations (e.g. COBE, IRAS, and surveys of large scale structures [10]). The idea, that a rule can exist among the fundamental constants, was presented by Dirac and by Eddington-Weinberg, but these rules were exact at Universe scale or subatomic scale. Here, a scale invariant rule is presented. Thanks to this relation the Universe appears self similar and its self similarity is governed by fundamental quantum quantities, like the Plank constant $h$, and relativistic constants, like the speed of light $c$.

Actually, there are some theories of gravity which are obtained from the Einstein-Hilbert gravitational action by adding scalar fields or curvature invariants of the form $\phi^{2} R, R^{2}, R_{\mu \nu} R^{\mu \nu}, R \square R$ [11, 12, [13. However, in the weak-limit approximation, all these theories fit very well with the experiments of Einstein's general relativity (tested only in this limit) 14. Moreover, the observations show a structure of Universe with scaling rules, where we can see globular clusters, single clusters or superclusters of galaxies, in which stars can be treated as massive point-like constituents of a universe mad of dust.

Why does the Universe appear with fixed scales, where matter can be clustered? The right question is not the previous one, but the following one: does the Universe have quantum nature at all scales? It appears that the Universe has a memory of its quantum origin like as suggested by R.Penrose with respect to quasi-crystal 15. Particularly, it is related to Penrose tiling and thus to $\varepsilon^{(\infty)}$ theory (Cantorian spacetime theory) as proposed by M.S. El Naschie [16], 17] 
as well as in A.Connes Noncommutative Geometry [18.

Some remarks are presented about the segregation of the Universe with respect to an Eddington-Weinberg-like relation $(h=m \sqrt{m G R}$, where $G$ is the Newton gravitational constant, $m$ is the mass of nucleon, $R$ is the radius of the Universe and $h$ is the quantum unit of action). In particular, we analyze the scale invariant law $R(N)=\frac{h}{M c} N^{\alpha}$, where $R$ is the radius of the astrophysical structures, $h$ is the Planck constant, $M$ is the total Mass of the self-gravitating system, $c$ the speed of light, $N$ the number of nucleons into the structures and $\alpha \simeq 3 / 2$. This relation is the Compton wavelength for $N=1$. The Newton gravitational constant $G$ probably plays no fundamental role in respect to the dimension of an object, while it becomes relevant in the interaction between the objects. So it is obvious that we have not found $G$ in the constitutive relations.

Another relevant point is the connection of the presented law with the Golden Mean. From the art to the science the role of the Golden Mean is well known 19. Here our expression agree with the Golden Mean and with the gross law of Fibonacci and Lucas 20.

The paper is organized as follows: we find the astrophysical scenario in Sec.2; Sec.3 presents a short review of definitions and properties for classic and stochastic self-similar random processes; Sec.4 is devoted to studying the exact determination of the power law at all significant scales and not only at astrophysical scales; in Sec.5 we briefly analyze some fundamental consequences from physical and geometric points of view and finally conclusions are drawn in Sec.6.

\section{Astrophysical scenario: quantum fluctuations and size of self-gravitating systems}

As it is known luminous matter appears segregated at different scale; in particular, we can distinguish among globular clusters, galaxies, clusters and superclusters of galaxies through their spatial dimensions [21], 22]. Table 1 recalls the dimensions and masses of previous systems [23], 24].

\begin{tabular}{|lll|}
\hline System Type & Length & Mass $\left(M_{\odot}\right)$ \\
Globular Clusters & $R_{G C} \sim 10 p c$ & $M_{G C} \sim 10^{6 \div 7}$ \\
Galaxies & $R_{G} \sim 1 \div 10 k p c$ & $M_{G} \sim 10^{10 \div 12}$ \\
Cluster of galaxies & $R_{C G} \sim 1.5 h^{-1} M p c$ & $M_{C G} \sim 10^{15} h^{-1}$ \\
Supercluster of galaxies & $R_{S C G} \sim 10 \div 100 h^{-1} M p c$ & $M_{S C G} \sim 10^{15 \div 17} h^{-1}$ \\
\hline
\end{tabular}

Table 1: Classification of astrophysical systems by length and mass, where $\mathrm{h}$ is the dimensionless Hubble constant whose value is in the range $[0.5,1]$.

In this paragraph, we consider systems where gravity is the only interaction among the constituents. For this reason, stars or objects smaller or larger than stars, where electromagnetic or nuclear interaction could be relevant, are not 
taken into account. Moreover, in the work only luminous matter is considered (dark matter will be considered in a future paper). Under these hypotheses, we can see stars as granular constituents of dust globular clusters or galaxies and so on. Moreover, a typical interaction length can be defined as a quantity which is proportional to the size of the system which contains the constituents. In other words, for each system we consider a maximum length, corresponding to its size, that plays the same role as the interaction length.

In 1965 Sakharov argued that quantum primordial fluctuations had to be related to cosmological evolution and to the dynamics of astrophysical systems [1. Eddington and later on Weinberg wrote the relevant relationship between quantum quantities and the cosmological ones:

$$
h \cong G^{1 / 2} m^{3 / 2} R^{1 / 2},
$$

where $h$ is the Plank constant, $G$ is the gravitational constant, $m$ is the mass of nucleon, and $R$ is the radius of Universe.

By following the Eddington-Weinberg (E-W) approach, we can write a general relationship between the radius $R$ of the self-gravitating system and its number of nucleons. While the E-W relationship was written only for the radius of Universe, we present a relationship which is scale invariant, so it can be adopted for all types of self-gravitating systems (and also for the entire universe).

It is interesting to note that if we write:

$$
R(N)=\frac{h}{M c} N^{\alpha},
$$

with $\alpha=3 / 2$, for $M=M_{G} \sim 10^{10 \div 12} M_{\odot}$ and $N=10^{68}$ (this is approximately the number of nucleons in a galaxy), we reproduce exactly $R \sim 1 \div 10 k p c$. In general, we can evaluate the number of nucleons in a self-gravitating system as

$$
N=M / m_{n},
$$

where $N$ is the number of nucleons of mass $m_{n}$ into self-gravitating system of total mass $M^{1}$. Then, we obtain the relevant results recalled in Table 2. In the second column the number of evaluated nucleons is shown, while we find the expected radius of self-gravitating system in the last column.

\begin{tabular}{|lll|}
\hline Sys Type & N.of Nucleons & Eval. Length \\
Glob. Clusters & $N_{G} \sim 10^{63 \div 64}$ & $R_{G C} \sim 1 \div 10 p c$ \\
Galaxies & $N_{G} \sim 10^{68}$ & $R_{G} \sim 1 \div 10 k p c$ \\
Cluster of gal. & $N_{C G} \sim 10^{72}$ & $R_{C G} \sim 1 h^{-1} M p c$ \\
Superc. of gal & $N_{S C G} \sim 10^{73}$ & $R_{S C G} \sim 10 \div 100 h^{-1} M p c$ \\
\hline
\end{tabular}

Table 2 : Evaluated Length for different self-gravitating systems

\footnotetext{
${ }^{1}$ In the present analysis the mass difference between proton and neutron is not relevant such as it will be shown below. The mass of nucleons is much larger than the mass of electrons, $m_{p}=1836 m_{e}$; therefore we can neglect the mass of electrons.
} 
By comparing the last column in Table 2 with the second column of Table 1 , we see a full agreement between the observed and theoretical values of the radius. It is obvious that if we have only one constituent (e.g. $N=1)$, like a proton or electron, the relation (2) is the standard and well-known Compton wavelength. Moreover, we have introduced the quantity $h / M c$ which is a type of Compton wavelength:

$$
\lambda_{M}=\frac{h}{M c} .
$$

It is interesting to note that at astrophysical scale we find a particular length. This allows us to obtain the exact radius of self-gravitation just by multiplying with the power of the number of nucleons which are present in the systems. We can make the following hypothesis: the observed universe appears self-similar to its quantum constituents. An invariant scale relation, from the quantum lengths to the astrophysical ones, plays a fundamental role. As macroscopic system, our universe shows a sort of quantum and relativistic memory of its primordial phase. The choice to start with $\alpha=3 / 2$ is suggested by statistical mechanics. Eq.(2) is strictly equivalent to

$$
R(N)=l \sqrt{N}
$$

where $l=h / m_{n} c$. Relation (2') is the well-known Random Walk equation or Brownian motion relation and it was firstly used by Eddington [6, 7], 8] .

In what follows we can observe that $\alpha=3 / 2$ is a too rough estimation if other interactions, in addition to gravity, are relevant. For this reason, we will consider stochastic self-similar processes at atomic scale. These processes generalize the classic ones. It is shown below that the nucleus scale is governed by a law like (2) but with a more complicated $l=l(N)$.

\section{Classic and stochastic self-similar random pro- cess}

Let $\Re$ be real space and $\gamma_{r} \in \Re^{+}$, then we define a self-similar (ss) random process for every $r>0$,

$$
X(s) \stackrel{d}{=} \gamma_{r} X(r s), \quad \text { with } \quad s \in \Re,
$$

where $\stackrel{d}{=}$ denotes equality as distributions [25].

The relation (5) is invariant under the group of positive affine transformations,

$$
X \rightarrow \gamma X, \quad s \rightarrow r s, \quad \gamma_{r}>0 .
$$

Since $\gamma_{r}$ satisfies the properties

$$
\gamma_{r_{1} r_{2}}=\gamma_{r_{1}} \gamma_{r_{2}}, \quad \forall r_{1}, r_{2}>0
$$


then it must have the form

$$
\gamma_{r}=r^{-\delta}, \quad \text { with } \quad \delta \in \Re .
$$

Thanks to (8) the relation (5) becomes

$$
X(s) \stackrel{d}{=} r^{-\delta} X(r s), \quad \text { with } \quad s \in \Re .
$$

When a process satisfies (5) or (9), it is said to be self-similar or $\delta$-self-similar.

A generalization of self-similar random process is obtained by replacing the deterministic scaling factor $\gamma_{r}=r^{-\delta}$ in (5) or (9) with a random variable $\widetilde{\gamma_{r}} \in \Re_{0}^{+}$. This variable is independent of the process to which such a variable is multiplied. Then eq.(9) becomes

$$
X(s) \stackrel{d}{=} \widetilde{\gamma_{r}} X(r s), \quad \text { with } \quad s \in \Re .
$$

D.Veneziano demonstrated in [26] that $\widetilde{\gamma_{r}}$ can also be written as $\gamma_{r}=r^{-\widetilde{\delta}}$ with $\widetilde{\delta}$ real random variable. Then, these kinds of processes, called stochastic selfsimilar (sss) random processes and the previous ones (ss), can be treated in the same theory. Gupta and Waymire showed that for $0<r \leq 1$ the sss processes are dilations, while for $r>1$ the sss processes are contractions [27], 28].

In [26] the author proved the following relevant theorem: if $\widetilde{\delta_{r_{1}}} \stackrel{d}{=} \widetilde{\delta_{r_{2}}}$ for some $r_{1} \neq r_{2}$, then $\widetilde{\delta}$ must be a deterministic constant $\delta$. Then, one can treat ss and sss random processes in a unique scheme.

Moreover, the author gives many relevant properties and generalizations to a $d$-dimensional space in the same paper, but we are not going to consider these properties because they do not fit the objectives of our paper (for more details see [26]).

Presently it appears clear there is an agreement between (5), (9) and (2), (2'). In fact, by defining the deterministic scaling parameter $\gamma_{r}=r^{-1 / 2}$, we find

$$
R(N)=\gamma_{r} R(r N)
$$

then our studies will explore a $1 / 2$-self-similar random aggregation process.

By considering electromagnetic and nuclear interactions, relation (9) becomes

$$
R(N)=\widetilde{\gamma_{r}} R(r N)
$$

with $\widetilde{\gamma_{r}}$ a random variable. In principle, we have to expect a change from a deterministic scaling parameter $(\gamma)$ to a random one $(\widetilde{\gamma})$, due to quantum treatment of nuclear and electromagnetic interactions.

\section{Exact determination of the power law coeffi- cient}

In this section, we invert the present point of view. We consider the mass and the radius of objects as known quantities and evaluate the power law respect 
to the observed data. We discover the validity of power law at all scales and not only at astrophysical scales. In other words, we obtain the impressive result that (2) is also correct at solar system, planet, human, organic cell and nucleon scales. Naturally, we have to expect a small difference in our model in respect to (2) due to the role of other physical interactions.

Let us consider the relation

$$
R(N)=\frac{h}{M c} N^{x},
$$

where $x$ is the quantity to be determined.

Then, we obtain

$$
x=\frac{\ln (R M / \alpha)}{\ln (N)},
$$

where $\alpha=h / c=2.2102209 \times 10^{-42} J s^{2} m^{-1}$. For the following evaluation,we are considering

$$
\begin{aligned}
1 p c & =3.085677587 \times 10^{16} \mathrm{~m}, \\
M_{\odot} & =1.98892 \times 10^{30} \mathrm{~kg}, \\
m_{p} & =1.6726231 \times 10^{-27} \mathrm{~kg} \\
h & =6.6260755 \times 10^{-34} \mathrm{Js} \\
c & =2.99792458 \times 10^{8} \mathrm{~ms}^{-1} .
\end{aligned}
$$

which are the well-known values reported in [29].

Table 3 summarizes the results in respect to the objects in the length range $10 p c<R<100 h^{-1} M p c$ and with a mass in the range $10{ }^{6 \div 7} M_{\odot}<M<$ $10^{17} h^{-1} M_{\odot}$. In particular, by following the data in table 1 , we obtain:

\begin{tabular}{|ll|}
\hline System Type & $x$ \\
Globular Clusters & $x_{G C}=1.5052 \div 1.5084$ \\
Galaxies (Giant) & $x_{G}=1.4975 \div 1.5273$ \\
Galaxies (Dwarf) & $x_{G}=1.5185 \div 1.5435$ \\
Cluster of galaxies & $x_{C G}=1.5185$ \\
Supercluster of galaxies & $x_{S C G}=1.5180 \div 1.5462$ \\
\hline
\end{tabular}

Table 3: Evaluated values of coefficient $x$ in power law for astrophysical objects.

From Table 3, we note that, in the first approximation, $x \simeq 1.5 \simeq 3 / 2$. This is also in a full agreement with the Fibonacci numbers and the Golden Mean trough the relation ${ }^{2} x=1+\phi$ with $\phi=\frac{\sqrt{5}-1}{2}$.

If we make the hypothesis that relation (13) is a universal law, then it has to be real at all scales. Due to other interactions (e.g electromagnetic and nuclear) the coefficient $x \sim 1.5$ and not exactly 1.5 . Table 4 summarizes the results in

\footnotetext{
${ }^{2}$ The connection was suggested by M.S.El Naschie in various pubblications [38.
} 
respect to solar system objects, while Table 5 is linked to organic matter and finally Table 6 is in respect to the nucleus of the periodic table of elements.

\begin{tabular}{|ccccc|}
\hline Object & Radius $\left(\mathbf{1 0}^{6} \mathbf{m}\right)$ & Mass $(\mathbf{K g})$ & $\mathbf{N}$ & $x$ \\
Sun & $6.96 \times 10^{2}$ & $M_{\odot}$ & $1.1892 \times 10^{57}$ & 1.4156 \\
Mercury & 2.439 & $3.2868 \times 10^{23}$ & $1.9650 \times 10^{50}$ & 1.4228 \\
Venus & 6.052 & $4.8704 \times 10^{24}$ & $2.9112 \times 10^{51}$ & 1.4209 \\
Earth & 6.378 & $5.976 \times 10^{24}$ & $3.5728 \times 10^{51}$ & 1.4206 \\
Mars & 3.3935 & $6.3943 \times 10^{23}$ & $3.8229 \times 10^{50}$ & 1.4233 \\
Jupiter & 71.4 & $1.8997 \times 10^{27}$ & $1.1358 \times 10^{54}$ & 1.4205 \\
Saturn & 59.65 & $5.6870 \times 10^{26}$ & $3.4000 \times 10^{53}$ & 1.4232 \\
Uranus & 25.6 & $8.6652 \times 10^{25}$ & $5.1806 \times 10^{52}$ & 1.4228 \\
Neptune & 24.75 & $1.0279 \times 10^{26}$ & $6.1453 \times 10^{52}$ & 1.4219 \\
Pluto & 1.1450 & $1.7928 \times 10^{22}$ & $1.0718 \times 10^{49}$ & 1.4270 \\
Moon & 1.738 & $7.3505 \times 10^{22}$ & $4.3946 \times 10^{49}$ & 1.4254 \\
\hline
\end{tabular}

Table 4: Calculated values of coefficient $x$ for solar system objects.

By considering Table 4 we note the impressive constancy of $x \sim 1.4$ for the planets of the solar system. The discrepancy of 0.1 , in respect to the expected value $\alpha=1.5$, could be an effect of the planets not being a self-gravitating system. For the Sun this discrepancy is a little bit worse than planets, probably due to not being a self-gravitating system and because of the effects of nuclear interactions in the interior of the Sun. The Moon, on the other hand, does not show a discrepancy worse than planets. As we know, the Moon is a second order system, not self-gravitating (e.g. there is a rotation around the Earth and another one around the Sun), but we found this is not relevant in the present study. In a recent paper, Lynden-Bell and Dwyer have derived from first physical principles a universal mass-radius relation for planets, white dwarfs and neutron stars [30. In the roughest approximation, the proposed mass-radius relation for planets reduces to $R \sim a_{0}\left(M / m_{p}\right)^{1 / 3}$ where $a_{0}=\hbar / m_{e} e^{2}$ which is equivalent to (2) when $\alpha=4 / 3$. Then, at this scale, a stochastic process is more appropriate than a self-similar one (as we will see in $\left(16^{\prime}\right)$ ). The aim of the present paper is not to obtain an accurate evaluation of the parameter of the solar system, but just to give a compatibility of our model with the solar system. A similar approach was recently presented in [31; also in this work the results reflect the Cantorian-fractal structure of the space-time. A detailed and interesting study on the quantization of the solar system was made by L.Nottale, G.Schumacher and J.Gay 32 .

At this point it is natural to test the validity of the hypothesis at organic scale. Table 5 summarizes the analysis on prokaryotic and eukaryotic cell and human organic matter. In respect to human scale, we have to take into account that a normal man is taller than he is wide; then we have to calculate an equivalent radius $R$. This radius is evaluated by considering the volume of a man as an equivalent volume on a sphere. In order to obtain the previous result 
we make the following considerations. Let us define

$$
\begin{array}{ll}
s & =\text { size } \\
c f r & =\text { circumference at half height }=2 \times s \\
h & =\text { height } \\
R^{\prime} & =\text { radius of } c f r \\
V^{\prime} & =\text { volume }=\pi\left(R^{\prime}\right)^{2} h \\
V & =\text { volume of the equivalent sphere }=4 / 3 \pi R^{3} \\
R & =\text { radius of the equivalent sphere }=\sqrt[3]{3 / 4\left(R^{\prime}\right)^{2} h}
\end{array}
$$

where the value $R$ is obtained thanks to the condition $V \prime=V$.

\begin{tabular}{|ccccc|}
\hline Organic Mat. & Radius $(\mathbf{m})$ & Mass(kg) & $\mathrm{N}$ & $x$ \\
Proc. cell & $10^{-6 \div-5}$ & $10^{-9} \div-8$ & $5.9786 \times 10^{17 \div 18}$ & $1.4729 \div 1.557$ \\
Euc. cell & $10^{-5 \div-4}$ & $1 \div 4 \times 10^{-7}$ & $2.3914 \times\left(2 \times 10^{19} \div 10^{20}\right)$ & $1.4847 \div 1.5557$ \\
Man & 0.3039 & 60 & $3.5872 \times 10^{28}$ & 1.5030 \\
\hline
\end{tabular}

Table 5: Calculated values of coefficient $\mathrm{x}$ for organic matter, where Prc. and Euc. stand for prokaryotic and eukaryotic.

To evaluate the last row of the Table 5 we have considered the following parameters:

$$
\begin{aligned}
s & =48 \text { (Italian size) } \\
c f r & =0.96 \mathrm{~m} \\
h & =1.60 \mathrm{~m} \\
R^{\prime} & =0.153 \mathrm{~m} \\
R & =\sqrt[3]{3 / 4\left(R^{\prime}\right)^{2} h}=0.3039 \mathrm{~m}
\end{aligned}
$$

Probably not everyone will agree with our previous choice. However, if we consider the case $R=h$ or $R=R \prime$, the $x$-values result in $x=1.53$ and $x=1.49$ respectively. In other words, our relation can give us the expected quantities also in organic matter. We can apply relation (2) in different ways to obtain relevant information about a fixed structure. For example, if we know the mass of an object we should be able to evaluate its radius, or when the radius is known we could evaluate its mass. It could be a good approach to get the dark matter in a structure by just considering fundamental quantities such as the Plank constant or the speed of light.

To conclude the analysis we have tested the hypothesis on the periodic table of the elements. The Universe, at all scale, is made with elements of the periodic table, then we have to determine the value of $x$ for all 103 elements. It is important to take into account the main part of the mass is in the nucleus; so, we have to consider this scale for estimating $R$. From nuclear physics we know 33 that

$$
R(A)=R_{0} A^{1 / 3},
$$

where $R_{0}=1.1 \div 1.5 \times 10^{-15} \mathrm{~m}$ and $A$ is atomic mass. Table 6 summarizes the obtained results ${ }^{3}$. We checked to see if the freedom of the choice of $R_{0}$ in the

${ }^{3}$ We have performed the analysis on all 103 elements, but only the first 26 elements (from $\mathrm{H}$ to $\mathrm{Fe}$ ) are reported here and we jump to the last one, i.e. Laurentium-Lr. 
range $1.1 \div 1.5$ is relevant and we found it is not; so we give only one value for $R$ in the table.

As a comment on Table 6, we can see that the value of the power law of $H e$ is the same value obtained for the planets of the solar system and the Sun. This is obvious! After the nucleosynthesis the ratio [n]/[p] is frozen (e.g. for $t \sim 1 \div 3$ min neutrons and protons link to produce deuterium nuclei, which create ${ }^{4} \mathrm{He}$ thanks to their fusion) 34. The fraction of primordial $\mathrm{He}$ in the aggregated matter must be the same. For these reasons the expectation of a value of $x$ for the planets and the Sun near $\mathrm{He}$ is natural. It is interesting to observe that $R_{0} \simeq h / m_{n} c=1.32 \times 10^{-15} \mathrm{~m}$. Relation (15) has a simple explanation: in a given nucleus, Heisenberg's relation of uncertainty implies that a nucleon occupies a volume $\sim(\Delta r)^{3}$ where $\Delta r \sim h / m_{n} c$ is of the order of Compton length of a nucleon. Consequently, the nucleus size must grow like

$$
R(N)=\frac{h}{M c} N^{4 / 3}=\frac{h}{m_{n} c} N^{1 / 3} .
$$

By using an sss process we obtain

$$
R(N)=\widetilde{\lambda_{M}} N^{\alpha}, \quad \text { with } \quad \alpha=3 / 2,
$$

where $\widetilde{\lambda_{M}}$ is the random function of the sss process with a formal expression $\widetilde{\lambda_{M}}=\frac{h}{M c} N^{-1 / 6}$. In other words, when other interactions act on a system and they are comparable or more relevant than the gravitational one, the aggregation process makes a transition from the ordinary self-similar process to the stochastic self-similar process.

Relation (16') is also in closer agreement with (2) and (15). A relevant point is the discrepancy of $\sim 0.08 \div 0.15$ among the elements in the periodic table with a value of $x \sim 1.35 \div 1.42$ and the theoretical value $\alpha=1.5$. In principle, we can consider the discrepancy as a consequence of the approximation $m_{n}=m_{p}$. This approximation is not relevant; in the worst case, that is, $L r$, we have $A-Z=260-103=157$ neutrons. By considering the difference $m_{n}-m_{p}=(939.56563-938.27231) \mathrm{MeV} / \mathrm{c}^{2}=1.2933 \times 10^{6} \mathrm{eV} / \mathrm{c}^{2}=2.3055 \times$ $10^{-30} \mathrm{~kg}$. In the case of Lr the correct mass becomes $M \prime=M_{0} * 103+\left(M_{0}+2\right.$. $\left.3055 \times 10^{-30}\right) * 157=431.96 \times 10^{-27} \mathrm{~kg}$; then we have a relative error $\sigma=$ $\frac{|M-M \prime|}{M}=\frac{\left|4.316 \times 10^{-25}-4.3196 \times 10^{-25}\right|}{4.316 \times 10^{-25}}=8.3411 \times 10^{-4}$. There is a correction in the coefficient $x$ of 0.001 , which is not relevant in the present analysis. For this reason the approximation $m_{n}=m_{p}$ is not a source of the discrepancy. Another possible reason could be due to the fact that we do not consider the mass of the electron. As it is known, $m_{p}=1836 m_{e}$, but also in this case, we find a relative error $\sigma^{\prime}=2.1528 \times 10^{-4}$ which is not significant. We can conclude that the only possible reason for the discrepancy is found in the presence of the other interactions (e.g. electromagnetic and nuclear interactions). In other words, the statistical geometry of the system is modified by other interactions. We find the trend of the power law coefficient $x$ as a function of the atomic number $Z$ in Fig. 1, while Fig. 3 shows the histogram for the 103 elements of the periodic table. 
From Fig. 1 it clearly appears that the ss-process is a rough approximation; in fact the elements are not on a constant line. This fact suggests a generalized scaling parameter like $\widetilde{\lambda_{M}}$ of the stochastic self-similar processes.

\begin{tabular}{|c|c|c|c|c|c|}
\hline Elements & $\mathrm{R}\left(10^{-15} \mathrm{~m}\right)$ & $\mathrm{A}$ & $\operatorname{Mass}\left(10^{-27} \mathrm{~kg}\right)$ & $\overline{\mathrm{N}}$ & $x$ \\
\hline$H$ & 1.5039 & 1.0079 & 1.6731 & 1 & - \\
\hline $\mathrm{He}$ & 2.3816 & 4.0026 & 6.6443 & 4 & 1.4199 \\
\hline$L i$ & 2.8613 & 6.9410 & 11.5220 & 7 & 1.3888 \\
\hline$B e$ & 3.1215 & 9.0122 & 14.9600 & 9 & 1.3884 \\
\hline$B$ & 3.3167 & 10.8100 & 17.9450 & 11 & 1.3734 \\
\hline$C$ & 3.4352 & 12.0110 & 19.9380 & 12 & 1.3818 \\
\hline$N$ & 3.6158 & 14.0067 & 23.2510 & 14 & 1.3787 \\
\hline$O$ & 3.7797 & 15.9994 & 26.559 & 16 & 1.3763 \\
\hline$F$ & 4.0025 & 18.9984 & 31.537 & 19 & 1.3738 \\
\hline $\mathrm{Ne}$ & 4.0837 & 20.179 & 33.497 & 20 & 1.3771 \\
\hline $\mathrm{Na}$ & 4. 2652 & 22.98977 & 38.163 & 23 & 1. 3712 \\
\hline$M g$ & 4. 345 & 24.305 & 40.346 & 24 & 1.3761 \\
\hline$A l$ & 4.499 & 26.98154 & 44.789 & 27 & 1. 3692 \\
\hline$S i$ & 4.5595 & 28.086 & 46.623 & 28 & 1.3703 \\
\hline$P$ & 4.7107 & 30.97376 & 51.416 & 31 & 1.3677 \\
\hline$S$ & 4.7652 & 32.06 & 53.22 & 32 & 1.3684 \\
\hline $\mathrm{Cl}$ & 4.9277 & 35.453 & 58.852 & 35 & 1.3717 \\
\hline$A r$ & 5.1277 & 39.948 & 66.314 & 40 & 1. 3652 \\
\hline$K$ & 5.0911 & 39.098 & 64.903 & 39 & 1.3668 \\
\hline $\mathrm{Ca}$ & 5.1333 & 40.08 & 66.533 & 40 & 1.3664 \\
\hline$S c$ & 5.3336 & 44.9559 & 74.627 & 45 & 1.3643 \\
\hline$T i$ & 5.4476 & 47.90 & 79.514 & 48 & 1.3634 \\
\hline$V$ & 5.5605 & 50.9414 & 84.563 & 51 & 1.3633 \\
\hline $\mathrm{Cr}$ & 5.5986 & 51.996 & 86.313 & 52 & 1.3635 \\
\hline$M n$ & 5.7023 & 54.9380 & 91.197 & 55 & 1.3627 \\
\hline $\mathrm{Fe}$ & 5.7336 & 55.847 & 92.706 & 56 & 1.362 \\
\hline$\cdots$ & $\begin{array}{c}\ldots \\
9.538\end{array}$ & $\begin{array}{c}\ldots \\
260\end{array}$ & $\begin{array}{c}\ldots \\
4316\end{array}$ & $\ldots$ & $\begin{array}{c}\cdots \\
3548\end{array}$ \\
\hline
\end{tabular}

Table 6: Calculated values of coefficient $\mathrm{x}$ for the periodic table of elements.

\section{Physical and geometrical consequences of the fundamental scale invariant law}

In the previous section we verified the validity of the (2) or (16') at all scales. Then, we considered the Compton wavelength expression as a particular case of a more general relation, one which is true for all material structures in the Universe. We discovered a fundamental relation which demonstrates the selfsimilarity of the Universe [38. We can evaluate the radius of a particular structure when the mass is known. The relations (2) and (16') show a Universe that 
has memory of its quantum and relativistic nature at all scales. In this sense, the Plank constant and the speed of light play a fundamental role in giving a quantum and relativistic parameterization of the structures. This reveals why the astrophysical structures and organic matter have their particular lengths 38 .

During the last twenty years much attention and strong research programs have been dedicated to the determination of Dark Matter. This is one of the most interesting problems of modern astrophysics. The presented scale invariant law can be used to evaluate the baryonic mass of the Universe. From (2) (with $\alpha=3 / 2^{4}$.) we have

$$
M_{U}=\left(\frac{R c}{h} m_{p}^{3 / 2}\right)^{2}=3.2841 \times 10^{55} \mathrm{~kg},
$$

which corresponds to a number of nucleons of

$$
N_{n u c l}=\frac{M_{U}}{m_{p}}=1.9634 \times 10^{82} .
$$

In the previous evaluation we considered a Universe with $R_{U}=6000 \mathrm{Mpc}=$ $1.8516 \times 10^{26} \mathrm{~m}$. Therefore, in a very rough approximation we can consider a spherical Universe where its density results in

$$
\rho_{U}=\frac{M_{U}}{4 / 3 \pi R_{U}^{3}}=1.219 \times 10^{-26} \mathrm{~g} / \mathrm{cm}^{3} .
$$

By introducing the critical density

$$
\rho_{c}=\frac{3 H^{2}}{8 \pi G}=2 \times 10^{-29} h^{2} \mathrm{~g} / \mathrm{cm}^{3},
$$

evaluated with $H=100 h \mathrm{~km} \mathrm{~s}^{-1} \mathrm{Mpc}^{-1}$ (where $0.5<h<1$ ), we found $\rho_{U}>$ $\rho_{c}$ which indicates a closed universe. The gravitational interaction is sufficient to reverse the expansion of the Universe into a contraction. This conclusion does not agree with the present observations. Moreover, the cosmological density becomes

$$
\Omega=\frac{\rho_{U}}{\rho_{c}}=\frac{9.8806 \times 10^{-27}}{2 \times 10^{-29}}=494.03
$$

which does not work.

Clearly the approximation of a spherical Universe is too rough. The self similarity of relation (2), and the exponent equal to $3 / 2$ are the two fundamental ingredients of fractal geometry. The scale invariant law lives in a fractal domain.

This means that Nature manifests itself trough its relativistic quantum fractal geometry aspects. The presented law has memory of its quantum origin through the Plank constant; of its relativistic origin thanks to the speed of light and of its fractal nature due to the fractal power law of nucleons. Therefore,

\footnotetext{
4 As we have seen this is the best value when gravity is the only relevant interaction.
} 
the Universe has fractal dimension. Following [35], 36], 37] we can define the fractal dimension as following

$$
D=\lim _{R \rightarrow \infty} \frac{\ln (N(<r))}{\ln (R)},
$$

where $N(<R)$ is the number of nucleons inside the radius $R$ and $R$ is the radius of the structure. Thanks to (21), we can estimate the fractal dimensions of all astrophysical structure and of the Universe too. Table 7 summarizes these results.

\begin{tabular}{|cc|}
\hline System Type & $D$ \\
Globular Clusters & $3.61 \div 3.66$ \\
Galaxies (Giant) & $3.27 \div 3.54$ \\
Galaxies (Dwarf) & $3.18 \div 3.39$ \\
Clusters of galaxies & 3.20 \\
Superclusters of galaxies & $2.94 \div 3.15$ \\
Universe & 3.13 \\
\hline
\end{tabular}

Table 7: Fractal Dimension of astrophysical objects

From Table 7 it is very interesting to note a relative coincidence of the fractal dimension of the Universe with the number $\pi$.

Taking into account the result

$$
D=3.1329
$$

it suggests a Universe whose spatial bound permeates the time dimension.

If we also consider the time, then

$$
D^{(4)}=4.1329 .
$$

Theoretically speaking by assuming the limitation of measurement accuracy the previous value can be the Hausdorff dimension, found by El Naschie 38

$$
D^{(4)} \cong\left\langle\operatorname{Dim} \varepsilon^{(\infty)}\right\rangle_{H}=4+\phi^{3}=4.236067977 .
$$

It is also interesting to note that $D^{(5)}=5.1329$, which is connected with the fine structure constant, i.e

$$
\left(D^{(5)}\right)^{3}=(5.1329)^{3} \cong \alpha_{0},
$$

as determined by El Naschie in [38.

The density of the Universe is:

$$
\rho_{U}^{\text {fractal }}=\frac{M_{U}}{4 / 3 \pi\left(R_{U}\right)^{D}}=2.1342 \times 10^{-30} \mathrm{~g} / \mathrm{cm}^{3},
$$


which is evaluated in the hypothesis of a spatial pseudo-sphere Universe (See Fig.3). A similar result can be reach by using a different approach based on the limit set of Klenian groups [39]. Therefore, $\rho_{U}<\rho_{c}$ indicates an open universe, i.e. the gravitational interaction is not sufficient to reverse the expansion of the Universe into a contraction. This conclusion fully agrees with the present observations 40, 41]. Moreover, the cosmological density results in

$$
\Omega=\frac{\rho_{U}}{\rho_{c}}=\frac{2.1342 \times 10^{-30}}{2 \times 10^{-29}}=0.11 .
$$

We may mention at this point that El Naschie in [38] used the dimensionless gravity constant $G$ to establish a shanon-like entropy $S(G)=\frac{\ln G}{\ln 2}+1=\bar{\alpha}_{e \omega} \simeq$ 128 where $\bar{\alpha}_{e w}$ is the coupling constant at the Higgs-Electroweak in order to establish quantum gravity.

\section{Conclusions}

In this paper we have discovered that the Compton wavelength relation is a particular case of a more general stochastic self-similar law. Our model allows us to realize an actual segregated universe according to the observations. Thanks to the relation $R=\lambda_{M} N^{\alpha}$, we have a link between the actual Universe, as observed, and its primordial phase, when quantum and relativistic laws were in comparison with gravity.

Relation (2) appears interesting not only because it allows us to obtain the exact dimensions of self-gravitating systems, but it is scale invariant. Moreover, it is extraordinary to obtain a single expression (see (16')) linking micro- and macro-universe in such a simple fashion. We have seen that this law is also valid for organic matter. Finally, the above relation is also exact in the presence of dark matter where we have more matter and a larger radius and it can be used again to set the quantities. If we consider $\alpha$ from nuclear physics $(\alpha=4 / 3)$, or the value for Hydrogen $\alpha_{H}$, we can evaluate the dark matter in the Universe or its related length. For these reasons it appears more general in respect to the questions posed in the present paper. There is no breaking point between microscopic and large scale universe thanks to the validity of (2) (or better $\left.\left(16^{\prime}\right)\right)$ at particles scale (traditional Compton wavelength). It is interesting to note that the observations on the large-scale structures and the Random Walk relation suggest $\alpha=3 / 2=1.5$ as best value (in agreement with El Naschie's E-infinity Cantorian spacetime, the Golden Mean and the Fibonacci numbers), while the nuclear physics relation (15) and our Planets scale results suggest $\alpha \sim 4 / 3=1.33$. Consequently, we could take into account that $\lambda_{M}$ can also be a random variable $\widetilde{\lambda_{M}}$, linked to the other interactions in addition to the gravitational interaction. Therefore, the self-similarity model has to be generalized by a stochastic self-similar model. This confirms the fractality of power law (2) which tends to be a more general theory (that is the theory of stochastic self-similar processes which we have just mentioned but the study of 
these kinds of processes is not the aim of our paper). In a certain sense, gravity was analyzed as a statistical property of space-time and the processes in it.

Our conclusion is that the fractal power law suggests a fractal Universe. Therefore, we can state that nature uses the language of a relativistic, quantum and fractal geometry.

\section{Acknowledgements}

The authors wish to thank S.Capozziello for suggestions.

\section{References}

[1] A. Sakharov, Zh.Eksp.Teor.Fiz 49, 245, 1965.

[2] L. Nottale, Fractal Space-Time and Microphysics: Towards a Theory of Scale Relativity, World Scientific, 1993.

[3] L. Nottale, Chaos, Solitons \& Fractals, 4, 361, 1994.

[4] L. Nottale, Chaos, Solitons \& Fractals, 6, 399-410, 1995.

[5] L. Nottale, Astron. and Astrophys. 327,867-889, 1997.

[6] M.S. El Naschie, On the unification of the fundamental forces and complex time in the $\varepsilon^{(\infty)}$ space, Chaos, Solitons and Fractals, 11, 1149-1162, 2000.

[7] B.J.Sidharth, Chaos Solitons Fractals 11, 2155, 2000; Chaos Solitons Fractals 12, 795, 2001.

[8] S.Capozziello et al., Mod.Phys.Lett. A16, 693, 2001.

[9] M. Rees, Our Cosmic Habitat, London: Weidenfeld and Nicolson, 2001.

[10] V.de Lapparent, M.J.Geller and J.P.Hucra, Astrophys.Jour. 302, L1, 1986. M.J.Geller and J.P.Hucra, Science 246, 897, 1989.

[11] D.La and P.J.Steinhardt, Phys.Rev.Lett.62, 376, 1989.

[12] I.L.Buchbinder, S.D.Odintsov, and I.L.Shapiro, Effective Action in Quantum Gravity, IOP Publishing, Bristol, 1992.

[13] K.S.Stelle, Phys.Rev.D 16, 953, 1977. Gen.Rev.Grav.9, 353, 1978.

[14] C.M.Will, Theory and Experiments in Gravitational Physics, Cambridge Univ.Press, Cambridge, 1993.

[15] R.Penrose, The Emperor's New Mind, Oxford University Press, 1989.

[16] M.S. El Naschie, On the uncertainty of Cantorian geometry and the two slit experiment, Chaos, Solitons and Fractals, 9(3), 517-529, 1998. 
[17] M.S. El Naschie, Penrose universe and Cantorian spacetime as a model for noncommutative quantum geometry, Chaos, Solitons and Fractals, 9, 931-933, 1998.

[18] A. Connes, Noncommutative Geometry, Academic Press, New York, 1994.

[19] T.A. Cook, The curves of life. Originally published by Constable and Company, London 1914; Reprinted by Dover Publications, New York. See also: M.S. El Naschie, Multidimensional Cantor-like Sets ergodic behaviour, Speculation in Science \& Technology, 15(2), 138-142, 1992.

[20] S. Vajda, Fibonacci and Lucas Numbers and the Golden Section, J.Wiley, New York, 1989.

[21] J.Binney and S.Tremaine, Galactic Dynamics, Princeton University Press, Princeton, 1987.

[22] B.A.Vorontsov-Vel'yaminov, Extragalactic Astronomy, Harwood Academic Pub, London, 1987.

[23] G.O.Abell, Astroph.Jou.S 3, 211, 1958.

[24] P.J.E.Peebles, Principles of Physical Cosmology, Princeton University Press, Princeton, 1993.

[25] W.Vervaat, Bull.Int.Statist.Inst. 52, 199, 1987.

[26] D.Veneziano, Fractals, Vol.7, No.1, 59, 1999.

[27] V.K.Gupta and E.C.Waymire, J.Geophys. Res. 95, 1999, 1990.

[28] V.K.Gupta et al., Water Resour.Res.95, 3405, 1994.

[29] Phys.Rev.D, Vol.54, N.1, Part 1, 1996; D.E. Groom et al., The European Physical Journal C15, 2000.

[30] D.Lynden-Bell and J.P.O'Dwyer, One mass-radiusrelation for Planets, White Dwarfs and Neutron Stars astro-ph/0104450

[31] M.Agop et al., $\varepsilon^{(\infty)}$ Cantorian space-time, polarization gravitational field and van der Waals-type forces, Chaos, Solitons and Fractals, 18, 1-16, 2003.

[32] L. Nottale, G.Schumacher, and J.Gay, Astron. and Astrophys. 322,10181025, 1997.

[33] W.Greiner and J.A.Maruhn, Nuclear Models, Springer Verlag, 1995.

[34] E.W.Kolb and M.S.Turner, The Early Universe, Addison-Wesley, NY, 1990.

[35] B. Mandelbrot B, The fractal Geometry of Nature, Freeman, NY, 1982. 
[36] F. Sylos Labini, M.Mountuori, L.Pietronero, Phys.Rep. 291, 1997.

[37] K.J.Falconer, Fractal Geometry, John Wiley and Sons, 1990.

[38] M.S. El Naschie, A review of E infinity theory and the mass spectrum of high energy partiche physics, to appear in Chaos, Solitons and Fractals, 2003.

[39] M.S. El Naschie, Klenian groups in $\varepsilon^{(\infty)}$ and their connection to particle physics and cosmology, Chaos, Solitons and Fractals, 16, 637-649, 2003.

[40] P.de Bernardis et al., Nature 404, 955, 2000.

[41] S.Masi et al., The BOOMERANG experiment and the curvature of the Universe, astro-ph/0201137, 2002. 